\title{
Evaluation of the safety and effects of pregabalin premedication on sleep quality, haemodynamic changes and pain in elderly patients with comorbidities undergoing spinal stenosis
}

\author{
Ebru Tarıkçı Kılıç,A-F( \\ ${ }^{1}$ Training and Research Hospital, Umraniye, Turkey \\ A - Research concept and design, B - Collection and/or assembly of data, C - Data analysis and interpretation, \\ $D$ - Writing the article, E - Critical revision of the article, F - Final approval of article
}

Ebru Tarıkçı Kılıç. Evaluation of the safety and the effects of pregabalin premedication on sleep quality, hemodynamic changes and pain in the elderly patients with comorbidities undergoing spinal stenosis. J Pre-Clin Clin Res. 2020; 14(4): 111-116. doi: 10.26444/jpccr/127924

\begin{abstract}
Objective. The aim of the study was to determine the effects of pregabalin premedication on preoperative sleep quality, intraoperative haemodynamic parameters, anesthesia consumption, blood loss, and postoperative pain control in patients over 65 years of age who had undergone surgery for spinal stenosis. The study also aimed to determine whether pregabalin premedication was safe for that age group and compare its effects with those of diazepam.

Materials and method. The retrospective study was conducted in a single centre after approval by the local Ethics Committee. The study sample consisted of 95 patients over 65 years of age who had undergone surgery for spinal stenosis. The sample was divided into two groups: Pregabalin premedication patients (PG; $n=45)$ and Diazepam patients $(D ; n=51)$. Demographic data, American Society of Anesthesiologists (ASA) scores, comorbidities, indications for operation, preoperative sleep quality, total anaesthesia consumption during surgery, intraoperative haemodynamic parameters and blood loss, time to leaving the post- anaesthetic care unit (Aldrete's > 9), postoperative visual analog pain scale (VAS), total fentanyl consumption via a patient-controlled analgesia (PCA) device, additional demand for analgesic, first mobilization, first oral intake, and side-effects were recorded.

Results. Participants did not significantly differ by demographic data and duration of surgery. The PG group had better sleep quality, lower systolic and diastolic blood pressure, heart rate, intraoperative bleeding, postoperative VAS scores, additional analgesic consumption, and total fentanyl consumption via a PCA, and earlier mobilization and oral intake than the D group. No serious side-effects were observed in the groups.

Conclusion. Pregabalin premedication is a safe and effective alternative medication for patients over 65 years of age.
\end{abstract}

Key words

Elderly, spinal stenosis, pregabalin, haemodynamic changes, intraoperative aaesthesia consumption, postoperative analgesia

\section{INTRODUCTION}

In industrialized countries, more than $10 \%$ of the elderly population are over the age of 85 . Aging causes an increase in the number of physiological changes in the systems and diseases. More than 35\% of elderly inpatients and 55\% of patients aged 65 and over undergo surgery. Geriatric premedication, therefore, becomes increasingly important in modern aaesthesia practice [1].

Stress and anxiety due to possible elective surgery cause a series of metabolic and hormonal changes in elderly patients (the pituitary-hypothalamic-adrenocortical system is stimulated, and the sympatho-adrenal pathway is activated) [2], resulting in an increase (up to $40 \%$ ) in the level of adrenaline [3]. Increased blood pressure and heart rate mean anaesthesia errors. Preoperative psychological status affects both intraoperative period and increases the need for a postoperative analgesic agent. Physiological changes with

Address for correspondence: Ebru Tarıkçı Kılıç, Training and Research Hospital, Umraniye, Turkey

E-mail: ebru.tarkc@yahoo.com

Received: 07.08.2020; accepted: 28.09.2020; first published: 07.10.2020 aging affect patients' response to anaesthesia. The doses of anaesthetic drugs for elderly patients should be carefully adjusted and even reduced when possible. Researchers are still looking for the best premedication agent for elderly patients and ways of administering it [3].

An ideal premedication agent should provide sedation, analgesia, and anxiolysis, increase the quality of induction, maintenance, and recovery, be administered orally, minimize the side-effects of the induction agent, and should not cause nausea, impair cardiovascular stability and suppress respiration [4]. Today, benzodiazepines, opioids, antihistamines, anticholinergics, alpha-2 adrenergics, antacids, and $\mathrm{H}_{2}$ antagonists are commonly used for premedication. New agents are expected to prevent anxiolytic, sedative, and amnestic effects and increase reflex activity, especially in elderly patients with a large number of comorbidities.

Postoperative pain varies according to the site and complexity of the surgical operation, causes physiopathological changes regardless of the surgical site and source, and delays recovery, and increases hospital stay and costs. The correct analgesic improves postoperative recovery and reduces hospital stay 
and medical costs. Woolf et al. (1913) were the first to argue that postoperative pain is a manageable acute pain that accompanies the inflammatory response to surgical trauma and becomes less intense with tissue recovery. Woolf laid groundwork for the emergence of the concept of 'preemptive analgesia' by using antinociceptive treatment that blocks the conduction of afferent signals. This technique is used to identify pain mechanisms to reduce pain due to incision and inflammation damage $[5,6]$.

Pregabalin is a GABA analog with no direct effect on GABA-like mechanisms. The analgesic actions of pregabalin establish an alpha-2 subunit to inhibit voltage-gated calcium channels, preventing neuronal depolarization and thereby reducing the release of excitatory neurotransmitters, resulting in pain control. Pregabalin has anticonvulsant, anxiolytic, and analgesic activity, and in recent years there has been an increase in the number of studies addressing its use for premedication [7].

This is the first study to investigate the safety of premedication pregabalin and its effect on preoperative sleep, intraoperative haemodynamic parameters, anaesthesia consumption, blood loss, and postoperative pain control in patients over 65 years of age who had undergone surgery for spinal stenosis, and also to compare its effects with those of diazepam.

\section{MATERIALS AND METHOD}

The sample consisted of 95 patients over 65 years of age, with an American Society of Anesthesiologists (ASA) physical status of I-III in a private single cenrer in 2015-16. The study was approved by the local Ethics Committee. A retrospective chart review design was used. Data were collected from anaesthesia registration forms, service nurse observation files, and the medical information system of the hospital. Preoperative records were used to collect data on participants' demographic characteristics, ASA scores, comorbidities, sleep quality measurements and indications for an operation. Intraoperative records were used to collect data on type of monitoring, total intraoperative anaesthesia consumption, haemodynamic parameters, blood loss, duration of surgery, and Aldrete scores. Postoperative records were used to collect data on VAS scores, postoperative analgesia consumption, additional opioid demand, first mobilization, first oral intake, and complications, if any.

The exclusion criteria were: 1) having an ASA score > III, 2) being followed-up in the postoperative intensive care unit, 3) allergic to the medications in question, 4) having renal and liver failure, (5) acute pancreatitis, (6) a history of pregabalin and gabapentin use, and (7) Alzheimer's and dementia. All participants underwent $5-8 \mathrm{ml} / \mathrm{kg} / \mathrm{h}$ balanced fluid replacement before anaesthesia.

The study sample was divided into two groups: pregabalin premedication group (PG; $\mathrm{n}=45)$ and diazepam group $(\mathrm{D} ; \mathrm{n}$ $=51$ ). The PG group received $300 \mathrm{mg}$ pregabalin (p.o) at 22:00 on the evening before surgery and two hours before surgery, while the $\mathrm{D}$ group received $10 \mathrm{mg}$ diazepam (p.o, tablet) at 22:00 on the evening before surgery and two hours before surgery. Participants' preoperative sleep quality, demographic characteristics, comorbidities, ASA scores, and surgical indications were evaluated and recorded. Routine ECG, non-invasive blood pressure, peripheral oxygen saturation, and Bispectral Index (BIS) were monitored before surgery. No additional intravenous premedication was performed before the induction of anaesthesia. All participants received $2 \mathrm{mg} /$ $\mathrm{kg}$ propofol (i.v) and $0.5 \mu \mathrm{g} / \mathrm{kg}$ fentanyl as anaesthetics, and $0.5 \mathrm{mg} / \mathrm{kg}$ rocuronium as the muscle relaxant. Mechanical ventilation (2lt/min with an oxygen/air ratio of 1:1) was administered following orotracheal intubation. Anaesthesia was maintained using propofol and remifentanil infusion $(40<$ BIS <50). Participants received an intermittent dose of rocuronium (i.v.) to provide muscle relaxation, and received $1,000 \mathrm{mg}$ paracetamol, $1 \mathrm{mg} / \mathrm{kg}$ tramadol and $8 \mathrm{mg}$ ondansetron (i.v) half an hour before surgery. They received no local anesthetic or additional analgesic or antiemetic (i.v) during surgery. An external heating system was used to prevent hypothermia during surgery. Remifentanil infusion was titrated to achieve non-invasive blood pressure $>30 \%$ of the baseline value. Participants received sugammadex to antagonize the effects of the muscle relaxant. Those with sufficient spontaneous breathing were extubated and then transferred to the recovery unit, and their vital signs were monitored until discharge criteria (Aldrete score of 9). Those with Aldrete $>9$ were transferred to the service.

After surgery, $100 \mathrm{ml}$ 0.9\% NACL containing $15 \mathrm{mcg} /$ $\mathrm{kg}$ fentanyl was administered via a PCA set at $2 \mathrm{~mL} / \mathrm{h}$ of continuous infusion dose and an increasing dose of $0.5 \mathrm{~mL}$ with a 15-min lockout interval. Haemodynamic parameters, total propofol and remifentanyl consumption, total bleeding, and duration of surgery were recorded during surgery. Total bleeding was measured by the total number of gauze pads used to absorb blood and the amount of blood in the aspirator. Postoperative pain was measured using the VAS scale (postop 2, 6, 12, and $24 \mathrm{~h}$ ). Participants with VAS $>6$ for $15 \mathrm{~min}$ in 24-hour period received $50 \mathrm{mg}$ tramadol for emergency analgesics and were recorded. The total amount of fentanyl administered via a PCA pump was calculated. Ramsay Sedation Scale (RSS) was used two hours after surgery for postoperative sedation evaluation.

The RSS scoring system describes 6 levels of sedation (from $1=$ anxious and agitated to $6=$ no response). RSS $>4$ indicated deep sedation. Dizziness, nausea, vomiting, and respiratory depression were evaluated as side-effects. Participants' sleep quality was evaluated using a table scored on a scale of $1-4$ ( 1 = best, 2 = good, 3 = medium, 4 = bad $)$.

All surgeries were performed by the same neurosurgeons and anaesthetists with more than 10 years of experience.

Statistical analysis. Frequency was used for nominal and ordinal data while means and standard deviations were used for scale data. The Kolmogorov-Smirnov test was used for normality testing. An independent samples t-test was used for normally distributed data while the Mann Whitney U test was used for non-normally distributed data. Data were analyzed using the Statistical Package for Social Sciences (SPSS for Windows, version 17) at a significance level of 0.05 .

\section{RESULTS}

Table 1 shows the participants' demographic characteristics, ASA scores, and comorbidities. There was no significant difference in age and body mass index (BMI) between the 2 groups. 
Table 1. Demographic data and baseline characteristics

\begin{tabular}{lccc}
\hline & Pregabalin $(\mathrm{n}=45)$ & Diazepam $(\mathrm{n}=51)$ & $\mathrm{p}$ \\
\hline Age & $72.64 \pm 3.63$ & $73.14 \pm 2.55$ & $0.139^{\mathrm{a}}$ \\
\hline BMI & $27.82 \pm 3.52$ & $27.20 \pm 2.46$ & $0.322^{\mathrm{a}}$ \\
\hline ASA scores, $\mathbf{n}(\%)$ & & & \\
\hline $\mathrm{II}$ & $14(31.1)$ & $29(56.9)$ & $0.001^{\mathrm{b}}$ \\
\hline III & $31(68.9)$ & $22(43.1)$ & $0.001^{\mathrm{b}}$ \\
\hline Comorbidities & & & \\
\hline HT, $\mathrm{n}(\%)$ & $24(53.3)$ & $24(47.1)$ & $0.539^{\mathrm{b}}$ \\
\hline Infectious diseases, $\mathrm{n}(\%)$ & $12(26.7)$ & $4(7.8)$ & $0.014^{\mathrm{b}}$ \\
\hline Malignancy, $\mathrm{n}(\%)$ & $18(40.0)$ & $23(45.1)$ & $0.614^{\mathrm{b}}$ \\
\hline Heart Failure, $\mathrm{n}(\%)$ & $6(13.3)$ & $8(15.7)$ & $0.744^{\mathrm{b}}$ \\
\hline COPD, $\mathrm{n}(\%)$ & $11(24.4)$ & $13(25.5)$ & $0.906^{\mathrm{b}}$ \\
\hline Others, $\mathrm{n}(\%)$ & $5(11.1)$ & $3(5.9)$ & $0.354^{\mathrm{c}}$ \\
\hline
\end{tabular}

A - Mann Whitney U Test; b - Chi-Square Test (Likelihood ratio); c - Chi-Square Test (Likelihood ratio)

BMI - Body Mass Index; ASA - American Society of Anesthesiologist; HT - Hypertension, COPD: Chronic obstructive pulmonary diseases.

There was also no significant differences in duration of surgery between the 2 groups. The PG group PG significantly lower intraoperative anaesthesia consumption (total propofol and remifentanyl) and total bleeding compared with group D $(\mathrm{p}<0.000)$. Disc extrusion was the most common indication for both groups. (Tab. 2). Comparison of the total blood loss is presented in Figure 1. Significantly total blood loss was lower in the PG group.

Table 2. Comparison of intraoperative findings and indications for operation

\begin{tabular}{|c|c|c|c|}
\hline & Pregabalin $(n=45)$ & Diazepam $(n=51)$ & $\mathrm{p}$ \\
\hline Duration of surgery (min) & $144.55 \pm 25.31$ & $143.43 \pm 23.14$ & $0.821^{\mathrm{b}}$ \\
\hline Propofol consumption (mg) & $5.04 \pm 0.56$ & $5.35 \pm 0.52$ & $0.009^{\mathrm{a}}$ \\
\hline Remifentanyl consumption (mg) & $9.82 \pm 0.39$ & $10.25 \pm 0.59$ & $0.000^{\mathrm{a}}$ \\
\hline Intraoperative blood loss (ml) & $391.00 \pm 172.45$ & $571.67 \pm 204.40$ & $0.000^{\mathrm{b}}$ \\
\hline \multicolumn{4}{|l|}{ Operation indications, $\mathbf{n}(\%)$} \\
\hline Neurodegenerative diseases & $5(11.1)$ & $4(7.8)$ & \multirow[t]{5}{*}{$0.842^{c}$} \\
\hline Extruding disc & $17(37.8)$ & $23(45.1)$ & \\
\hline Scoliosis & $2(4.4)$ & $4(7.8)$ & \\
\hline Tumour & $15(33.3)$ & $15(29.4)$ & \\
\hline Trauma & $6(13.3)$ & $5(9.8)$ & \\
\hline
\end{tabular}

a ${ }^{a}$ Mann Whitney U Test; ${ }^{\mathrm{b}}$ - Independent Samples T-Test; ${ }^{\mathrm{c}}$ - Chi-Square Test (Likelihood ratio)

Table 3 shows the participants' pre- and post-induction systolic blood pressure, diastolic blood pressure, and maximum heart rate values ( $30 \mathrm{~min}, 1 \mathrm{~h}$, and $2 \mathrm{~h}$ ) and VAS scores. The group PG had significantly lower scores in all measurements than the group D $(\mathrm{p}<0.05)$ (Fig. 2).

In the postoperative period, the recorded total fentanyl consumption from PCA was found to be higher in the D group, although it was not statistically significant. Aldrete score, which was the most important criteria in terms of discharge to the service, was higher in the PG group. Sleep quality was better in the PG group and anxiety was found to be lower which we believed seconder to the sleep quality. In addition to these finding, early mobilization and first oral intake were significantly faster in the PG group (Tab. 4)

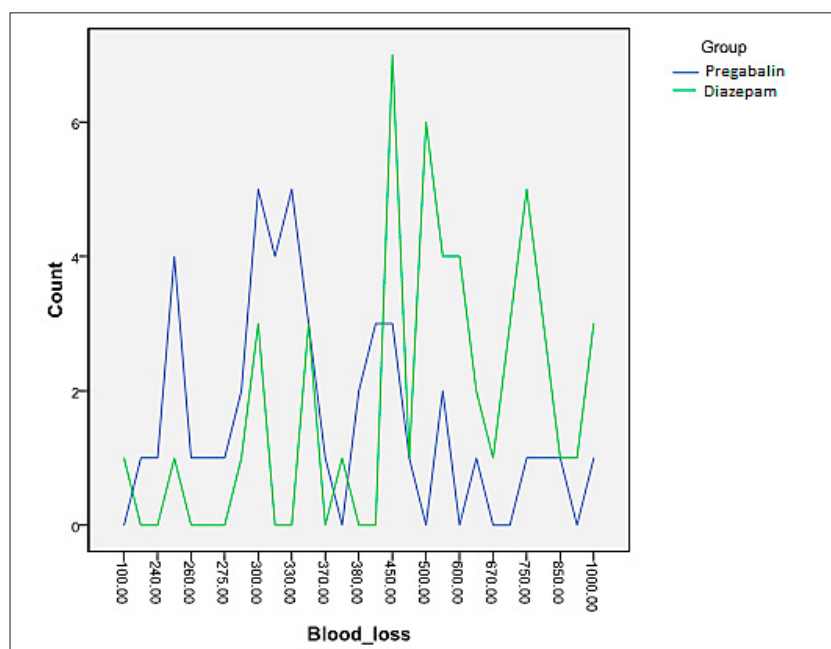

Figure 1. Blood loss

Table 3. Comparison of time-dependent measurements and difference analysis results

\begin{tabular}{lccc}
\hline & Pregabalin $(\mathrm{n}=45)$ & Diazepam $(\mathrm{n}=51)$ & $\mathrm{p}$ \\
\hline Pre-induction SBP & $138.78 \pm 13.12$ & $158.98 \pm 21.33$ & $0.000^{\mathrm{a}}$ \\
\hline Pre-induction DBP & $68.56 \pm 9.03$ & $75.65 \pm 13.19$ & $0.013^{\mathrm{b}}$ \\
\hline Post-induction SBP & $99.80 \pm 9.21$ & $143.94 \pm 20.21$ & $0.000^{\mathrm{b}}$ \\
\hline Post-induction DBP & $56.42 \pm 5.55$ & $68.39 \pm 11.20$ & $0.000^{\mathrm{b}}$ \\
\hline Systolic 30 min & $108.02 \pm 10.49$ & $131.69 \pm 18.28$ & $0.000^{\mathrm{a}}$ \\
\hline Diastolic 30 min & $61.60 \pm 6.72$ & $63.75 \pm 9.85$ & $0.211^{\mathrm{a}}$ \\
\hline Systolic 1 h & $105.07 \pm 13.21$ & $125.96 \pm 16.12$ & $0.000^{\mathrm{a}}$ \\
\hline Diastolic 1 h & $59.04 \pm 8.06$ & $61.82 \pm 9.17$ & $0.120^{\mathrm{a}}$ \\
\hline Systolic 2 h & $105.91 \pm 11.97$ & $122.61 \pm 14.47$ & $0.000^{\mathrm{a}}$ \\
\hline Diastolic 2 h & $58.40 \pm 6.08$ & $59.20 \pm 6.61$ & $0.573^{\mathrm{b}}$ \\
\hline Pre-HR & $76.63 \pm 12.47$ & $76.87 \pm 10.24$ & $0.659^{\mathrm{b}}$ \\
\hline Post-HR & $72.38 \pm 7.08$ & $75.53 \pm 10.38$ & $0.083^{\mathrm{a}}$ \\
\hline HR 30 min & $72.42 \pm 6.79$ & $76.86 \pm 10.95$ & $0.095^{\mathrm{b}}$ \\
\hline HR 1 h & $71.80 \pm 5.03$ & $76.45 \pm 9.53$ & $0.003^{\mathrm{a}}$ \\
\hline HR 2 h & $74.53 \pm 5.19$ & $76.92 \pm 9.03$ & $0.111^{\mathrm{a}}$ \\
\hline VAS pre & $4.44 \pm 1.06$ & $5.67 \pm 0.93$ & $0.000^{\mathrm{b}}$ \\
\hline VAS 2h & $3.24 \pm 0.74$ & $3.98 \pm 0.99$ & $0.000^{\mathrm{b}}$ \\
\hline VAS 6h & $2.62 \pm 0.65$ & $3.08 \pm 1.00$ & $0.019^{\mathrm{b}}$ \\
\hline VAS 24h & $2.16 \pm 0.71$ & $2.86 \pm 0.95$ & $0.000^{\mathrm{b}}$ \\
\hline
\end{tabular}

A - Independent Samples T-Test; b - Mann Whitney U Test.

SBP - Systolic Blood Pressure; DBP - Diastolic Blood Pressure; HR - Heart rate; VAS - Visual Analog scale.

Additional opioid requirement was found to be higher in the D group. When the side- effects were checked, nausea vomiting dizziness were observed more frequently in the $\mathrm{D}$ group during the postoperative period (Tab. 5).

\section{DISCUSSION}

Pregabalin is a new generation antiepileptic that reduces the release of neurotransmitters such as glutamate, noradrenaline, and substance P. A daily 150-600 mg dose of pregabalin has been approved in Turkey as an additional treatment for adult patients with peripheral neuropathic pain, 


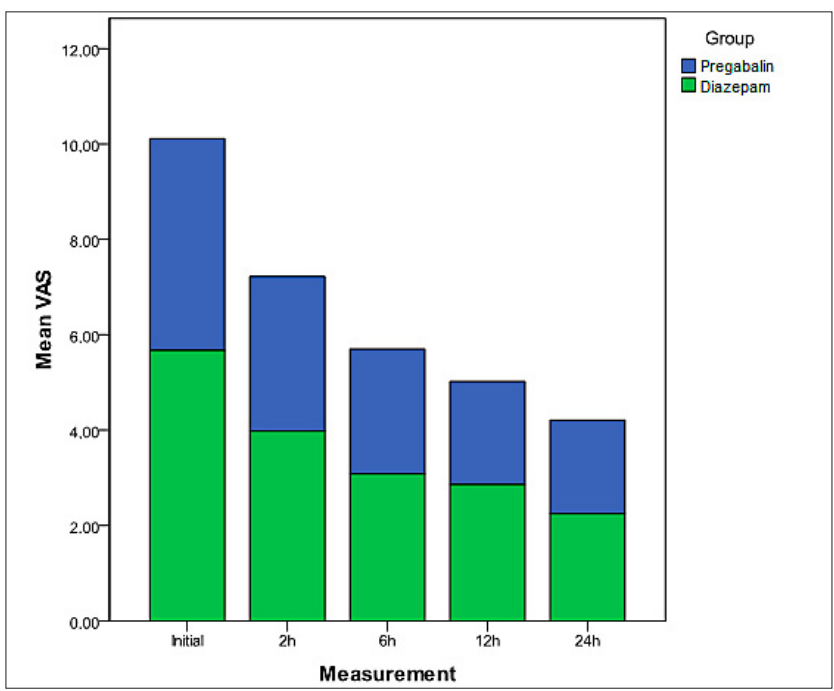

Figure 2. Mean VAS values

Table 4. Group outcomes and difference analysis results

\begin{tabular}{lccc}
\hline & Pregabalin $(\mathrm{n}=45)$ & Diazepam $(\mathrm{n}=51)$ & $\mathrm{p}$ \\
\hline Total fentanyl consumption PCA & $17.47 \pm 0.73$ & $\mathbf{1 7 . 5 1 \pm 0 . 8 6}$ & $0.691^{\mathrm{a}}$ \\
\hline Aldrete scores & $\mathbf{1 1 . 4 1 \pm \mathbf { 1 . 9 9 }}$ & $6.71 \pm 1.27$ & $0.000^{\mathrm{a}}$ \\
\hline Sleep Quality & $1.24 \pm 0.57$ & $\mathbf{2 . 4 5} \pm \mathbf{0 . 7 3}$ & $0.000^{\mathrm{a}}$ \\
\hline RSS at 2 h & $2.07 \pm 0.25$ & $\mathbf{2 . 1 4} \pm \mathbf{0 . 3 5}$ & $0.261^{\mathrm{a}}$ \\
\hline Additional opioid demand, $\mathrm{n}(\%)$ & $9(20.0)$ & $\mathbf{4 1 ( 8 0 . 4 )}$ & $0.000^{\mathrm{b}}$ \\
\hline Preop anxiety & $46.20 \pm 3.98$ & $46.65 \pm 5.74$ & $0.041^{\mathrm{a}}$ \\
\hline First mobilization & $8.62 \pm 0.49$ & $9.84 \pm 0.97$ & $0.000^{\mathrm{a}}$ \\
\hline First oral intake & $5.20 \pm 0.99$ & $6.98 \pm 1.14$ & $0.000^{\mathrm{a}}$ \\
\hline
\end{tabular}

a - Mann Whitney U Test; ${ }^{\mathrm{b}}$ - Chi-Square Test

Table 5. Postoperative side effects or complications

\begin{tabular}{lccc}
\hline & Pregabalin $(\mathrm{n}=45)$ & Diazepam $(\mathrm{n}=51)$ & $\mathrm{p}$ \\
\hline Nausea, $\mathrm{n}(\%)$ & $7(15.6)$ & $\mathbf{9 ( 1 7 . 6 )}$ & $0.784^{\mathrm{a}}$ \\
\hline Vomiting, $\mathrm{n}(\%)$ & $4(8.9)$ & $\mathbf{9 ( 1 7 . 6 )}$ & $0.211^{\mathrm{a}}$ \\
\hline Dizziness, $\mathrm{n}(\%)$ & $2(3.9)$ & $\mathbf{1 1 ( 2 4 . 4 )}$ & $0.784^{\mathrm{a}}$ \\
\hline
\end{tabular}

${ }^{a}$ Chi-Square Test

diffuse anxiety disorder, fibromyalgia, and partial epilepsy. It is a rapid-acting anxiolytic and analgesic that achieves maximum plasma concentration approximately one hour after oral intake. Preemptive administration of pregabalin results in the blockage of the neuropathic component of acute nociceptive surgical pain, thereby reducing chronic neuropathic pain, opioid demand, pre-and postoperative anxiety and stress $[8,9]$. Pregabalin may also play a significant role in postoperative pain management because it reduces excessive excitability of dorsal horn neurons induced by tissue damage, and inhibits hyperalgesia and central sensitivity [10]. There are few studies that investigate the effects of preemptive pregabalin on pain and analgesic consumption. What is more, they report conflicting results due to differences in dosage regimen and type of surgery. Therefore, further research is warranted.

In the current study, administration of $300 \mathrm{mg}$ pregabalin (p.o) the night before the surgery enabled the participants to have better sleep, which is considered to play a secondary role in anxiety reduction. Administration of $300 \mathrm{mg}$ pregabalin (p.o) on the morning of the surgery enabled the PG group to have more stable haemodynamic values and less bleeding, which is secondary to persistent balanced hypotensive anaesthesia throughout the surgery than the D group. In the postoperative period, the PG group had lower VAS scores and opioid consumption, earlier mobilization (with the reduction of pain), lower urgent demand for opioid than the group D. Adult patients with increased preoperative anxiety had significantly more postoperative pain and a higher demand for analgesic and a sedative drug. Moreover, the less the anxiety in the preoperative period, the faster the recovery.

Although a large number of premedication drugs has been developed in recent years, more than $80 \%$ of patient still report postoperative anxiety and pain. Gonano et al. [11] preoperatively administered $300 \mathrm{mg}$ pregabalin to 40 patients and reported low anxiety in patients after orthopedic surgical interventions. Polat et al. (8) found that $150 \mathrm{mg}$ pregabalin reduced post abdominal hysterectomy anxiety in patients, and therefore recommend it as an alternative premedication. Omara AF et al. [12] reported that $150 \mathrm{mg}$ preemptive pregabalin significantly reduced the demand for urgent postoperative analgesics and improved the sleep quality of patients on the first night after femur fixation. Luo et al. [13] reported that patients with preoperative sleep quality had less severe postoperative pain.

Singh et al., [14] who compared pregabalin premedication and placebo, found that pregabalin inhibited stress response to laryngoscopy and intubation, resulting in stable perioperative haemodynamic parameters. Eren et al. [15] showed that 50 patients who underwent elective lumbar microdiscectomy surgery and received pregabalin had lower mean arterial pressure and heart rate values, and lower postintubation tachycardia and hypertension. Chakraborty [16] and Sundar [17] conducted research on pregabalin at different times and obtained similar results for $150 \mathrm{mg}$. Saxena et al. [18] compared 150 and $300 \mathrm{mg}$ doses of pregabalin, and reported no difference in laryngoscopy and intraoperative haemodynamic parameters between the 2 groups. Reuben et al. [19] divided patients scheduled for lumbar laminectomy into 4 groups; 1) placebo, 2) $400 \mathrm{mg}$ celecoxib, (3) $150 \mathrm{mg}$ pregabalin, and 4) $150 \mathrm{mg}$ pregabalin $+400 \mathrm{mg}$ celecoxib one hour before and 12 hours after surgery. They concluded that pre- and postoperative pregabalin was as effective in pain relief and morphine consumption as celecoxib, and that the combination of both drugs was the most effective treatment. Spreng et al. [20] divided 50 patients scheduled for discectomy into 2 groups: 1) $150 \mathrm{mg}$ oral pregabalin one hour before surgery and 2) placebo. They concluded that the pregabalin group had significantly lower pain scores and opioid consumption than the placebo group. Kim et al. [21] sought an effective dose of preoperative oral pregabalin for the multi-model analgesia regimen in lumbar spinal fusion surgery and concluded that the $150 \mathrm{mg}$ pregabalin group had less opioid consumption and demand for it. Researchers have used different doses of preemptive pregabalin for hysterectomy, spinal surgery, orthopedic surgery, and laparoscopic surgery. Baloyiannis et al.[22] investigated the effects of $300 \mathrm{mg}$ pregabalin administered before laparoscopic colorectal surgery on postoperative morphine consumption and concluded that those who received pregabalin had significantly lower morphine consumption on 8, 24 and 48 hours than the placebo group. 
In 2017, $\mathrm{Hu}$ Jiai conducted a literature research on studies about pregabalin and gabapentin premedication and included 79 randomized controlled trials with 6,201 patients who received single dose premedication. The conclusion was that there was a dose response relationship between opioid consumption and postoperative pain (24). A similar study was designed by Gupta et al. to evaluate the effect of pregabalin as premedication on the perioperative anesthetic requirement and analgesia. They recommended premedication with $75 \mathrm{mg}$ of pregabalin the night before and $300 \mathrm{mg}$ an hour before surgery as this decreased analgesic consumption in the postoperative period without causing any adverse effects (25). Rajappa conducted a randomized controlled study on 135 patients under spinal anaesthesia and concluded that premedication with $150 \mathrm{mg}$ of pregabalin provided postoperative pain relief (26).

\section{RESULTS}

Massive bleeding during spinal stenosis surgeries adversely affects intraoperative and postoperative haemodynamics, especially in elderly patients (vision in the surgical field, difficulty of intervention, hemodynamic problems). In the current study, balanced and controlled hypotensive anaesthesia resulted in reduced intraoperative bleeding in the participants.

The PG group had significantly earlier mobilization than the D group, which may have been due to low VAS scores. Warner FM et al. [23] employed an experimental animal model and reported that pregabalin improved motor recovery on months $1,3,6$, and 12 in spinal cord injury. Considering this study, further research on spinal surgeries is therefore warranted.

The incidence of nausea and vomiting was significantly lower in the PG group participants, who had an earlier oral intake than group D participants. This is an important result because early oral feeding promotes recovery. Due to the residual effect of pregabalin, the PG group also had longer anaesthesia recovery and higher postoperative RRS than the D group. Long early postoperative sedoanalgesia and lack of stable hemodynamics and respiratory depression deficiency is, in a sense, ideal.

Pregabalin is a drug with a low frequency of side-effects, little interaction with other drugs, and good oral intake tolerance, although there are research reports on side-effects such as drowsiness, dizziness, dry mouth, and somnolence. In the current study, participants reported dizziness and dry mouth. Previous studies have also reported an increase in dose-related side-effects. The results obtained in this study show that $300 \mathrm{mg}$ preemptive pregabalin is safe for elderly patients.

\section{CONCLUSIONS}

Two doses of $300 \mathrm{mg}$ of premedication pregabalin for spinal stenosis surgery resulted in high sleep quality, low intraoperative anaesthesia and analgesic consumption, low postoperative pain score, low demand for emergency rescuing analgesics, early mobilization and early oral intake, which are parameters that play a significant role in recovery. Premedication pregabalin did not cause any serious sideeffects in patients over 65 years of age.
This study has one limitation: it was based on a retrospective design. Further randomized controlled studies with larger sample sizes are needed to provide more evidence regarding the effects of premedication pregabalin. In conclusion, it is believed that premedication pregabalin is a good alternative adjuvant in the elderly.

\section{Compliance with ethical standards}

All procedures were in accordance with the ethical standards of the institutional and national research committee and with the 1964 HelsinkiDdeclaration and its later amendments.

\section{Funding}

No financial or support was received from any pharmaceutical company that had a direct connection with the research.

\section{Conflict of interest}

The authors declare that they have no conflicts of interest.

\section{REFERENCES}

1. Ayşin Ersoy, Menşure Yılmaz Çakırgöz, Ülkü Aygen Türkmen. Geriatric Anesthesia. Okmeydanı Medical Journal. 2013; 29(2): 106-109.

2. Kowark A, Rossaint R, Keszei AP, Bischoff P, Czaplik M, Drexler B, et al. Impact of Preoperative Midazolam on Outcome of Elderly patients (I-PROMOTE): study protocol for a multicentre randomised controlled trial. Trials. 2019 Jul 15; 20(1): 430.

3. Wolfe JD1, Wolfe NK1, Rich MW1. Perioperative care of the geriatric patient for noncardiac surgery. Clin Cardiol. 2020 Feb; 43(2): 127-136. doi: $10.1002 /$ clc. 23302

4. Andres TM1, McGrane T2, McEvoy MD3, Allen BFS4. Geriatric Pharmacology: An Update. Anesthesiol Clin. 2019 Sep; 37(3): 475-492. 5. Nabavighadi K, Batista C, Ghoddoussi F, Kumar N, Aiello A, Reeves B, Krishnan S, Ellis T 2nd. Oral multimodal preemptive analgesia improves postoperative pain control and decreases opioid utilization in spinal fusion patients. J Clin Anesth. 2020 May; 61: 109679.

6. Woolf CJ, Chong MS. Preemptive analgesia - treating postoperative pain by preventing the establishment of central sensitization. Anesth Analg. 1993 Aug; 77(2): 362-79.

7. Sills GJ, Rogawski MA. Mechanisms of action of currently used antiseizure drugs. Neuropharmacology. 2020 May 15; 168: 107966.

8. Polat Bora S, Kol Özdemir İ, Avcı O, İşbir A, Kaygusuz K, Gürsoy S. Evaluation of Preoperative Anxiolytic and Postoperative Analgesic Effects of Pregabalin Premedication. Turkiye Klinikleri J Med Sci. 2019; 39(1): 9-18.

9. Karube N, Ito S, Sako S, Hirokawa J, Yokoyama T. Sedative effects of oral pregabalin premedication on intravenous sedation using propofol target-controlled infusion. J Anesth. 2017 Aug; 31(4): 586-592.

10. Christopher W, Goodman Allan S Brett. Gabapentin and Pregabalin for Pain - Is Increased Prescribing a Cause for Concern? N Engl J Med 2017; 377: 411-414.

11. Gonano C, Latzke D, Sabeti-Aschraf M, Kettner SC, Chiari A, Gustorff $B$. The anxiolytic effect of pregabalin in outpatients undergoing minor orthopaedic surgery. J Psychopharmacol. 2011 Feb; 25(2): 249-51.

12. Amany F Omara, Sameh A Ahmed, Motaz MA Abusabaa. The Effect Of The Use Of Pre-Emptive Oral Pregabalin On The Postoperative Spinal Analgesia In Patients Presented For Orthopedic Surgeries: Randomized Controlled Trial. J Pain Res. 2019; 12: 2807-2814.

13. H Luo Z-Y Feng. A review of pregabalin in the treatment of postoperative pain. Chinese Journal of new drugs 22(8): 915-918.

14. Dheer Singh, Jaybrijesh Singh Yadav, Birendra Kumar Jamuda, and Pooja Singh. Oral Pregabalin as Premedication on Anxiolysis and Stress Response to Laryngoscopy and Endotracheal Intubation in Patients Undergoing Laparoscopic Cholecystectomy: A Randomized DoubleBlind Study. Anesth Essays Res. 2019 Jan-Mar; 13(1): 97-104.

15. Gülay EREN, Betül KOZANHAN, Oya HERGÜNSEL, Ünsal BİLGİN, Güray DEMİR, Zafer ÇUKUROVA. Pregabalin Blunts Cardiovascular Response to Laryngoscopy and Tracheal Intubation. Turkiye Klinikleri J Anest Reanim. 2009; 7(2): 82-7.

16. Chakraborty R, Jain R, Sharma R. Evaluation of the efficacy of preoperative oral pregabalin in attenuating hemodynamic response to 
laryngoscopy and intubation and on post-operative pain in patients undergoing elective surgery under general anaesthesia. Indian J Clin Anaesthe. 2016; 3: 419-26.

17. Sundar AS, Kodali R, Sulaiman S, Ravullapalli H, Karthekeyan R, Vakamudi M, et al. The effects of preemptive pregabalin on attenuation of stress response to endotracheal intubation and opioid-sparing effect in patients undergoing off-pump coronary artery bypass grafting. Ann Card Anaesth. 2012; 15: 18-25.

18. Saxena A, Gupta P, Chaudhary L. Effect of pregabalin premedication on the laryngoscopy response and intra-operative hemodynamic variables in laparoscopic cholecystectomy: A randomized comparison of two doses. Int J Sci Stud. 2016; 4: 75-80.

19. Reuben SS, Buvanendran A, Kroin JS, Raghunathan K. The analgesic efficacy of celecoxib, pregabalin, and their combination for spinal fusion surgery. Anesth Analg. 2006; 103: 1271-1277.

20. Spreng UJ, Dahl V, Raeder J. Effect of a single dose of pregabalin on post-operative pain and pre-operative anxiety in patients undergoing discectomy. Acta Anaesthesiol Scand. 2011; 55(5): 571-576.

21. Kim SY, Song JW, Park B, Park S, An YJ, Shim YH. Pregabalin reduces post-operative pain after mastectomy: A double-blind, randomized, placebo-controlled study. Acta Anaesthesiol Scand. 2011; 55: 290-6.
22. Baloyiannis I, Theodorou E, Sarakatsianou C, Stavroula G, Periyoliotis K, Tzovaras G. The effect of preemptive use of pregabalin on postoperative morphine consumption and analgesia levels after laparoscopic colorectal surgery: a controlled randomized trial. International Journal of Colorectal Disease. 2020; 35(2): 323.

23. Warner FM, Cragg JJ, Jutzeler CR, Röhrich F, Weidner N, Saur M, Maier DD, Schuld C; EMSCI Sites, Curt A, Kramer JK. Early Administration of Gabapentinoids Improves Motor Recovery after Human Spinal Cord Injury. Cell Rep. 2017 Feb 14; 18(7): 1614-1618.

24. Hu J, Huang D, Li M, Wu C, Zhang J. Effects of a single dose of preoperative pregabalin and gabapentin for acute postoperative pain: a network meta-analysis of randomized controlled trials. J Pain Res. 2018; 11: 2633-2643.

25. Prasoon Gupta, Anudeep Saxena, Lalita Chaudhary. Effect of Pregabalin Premedication on the Requirement of Anesthetic and Analgesic Drugs in Laparoscopic Cholecystectomy: Randomized Comparison of Two Doses. Anesth Essays Res. 2017 Apr-Jun; 11(2): 330-333.

26. Rajappa GC, Vig S, Bevanaguddaiah Y, Anadaswamy TC. Efficacy of Pregabalin as Premedication for Post-Operative Analgesia in Vaginal Hysterectomy. Anesth Pain Med. 2016; 6(3): e34591. 